\title{
Aspectos fitoquímicos e potencialidades biológicas da Crescentia: uma revisão narrativa
}

\author{
Phytochemical aspects and biological potentials of Crescentia: a narrative review
}

Aspectos fitoquímicos y potenciales biológicos de Crescentia: una revisión narrativa

Viviane da Silva Lima ${ }^{1 *}$, Raíssa da Conceição Santos ${ }^{1}$, Maria Lúcia Henrique Ramos ${ }^{1}$, Renato Elói da Silva ${ }^{1}$, João Victor do Nascimento Nunes ${ }^{1}$, Renata Magalhães Ramos ${ }^{1}$, Jamilly Moura Feitosa ${ }^{1}$, Lydja Rayhanne Dário Ferreira' ${ }^{1}$, Jessica da Silva Siqueira ${ }^{2}$, Gabriela Cavalcante da Silva ${ }^{1,3}$.

\section{RESUMO}

Objetivo: Revisar e compilar informações descritas na literatura sobre a composição química e atividades biológicas do gênero Crescentia. Revisão bibliográfica: A Crescentia cujete L. mostrou ser tão eficaz quanto Crescentia alata kunt em relação aos efeitos terapêuticos. As espécies possuem diversos compostos bioativos isolados e identificados através de técnicas espectrométricas, compostos estes, ligados às atividades biológicas apresentadas nas espécies do gênero, até mesmo as atividades que necessitam de relativa toxicidade, como atividades antimicrobiana e anticancerígena, destacando-se como uma futura candidata a fornecer seus componentes para a indústria farmacêutica. Muitas espécies são utilizadas, no uso popular, de forma inadequada, tendo em vista que grande parte dos usuários não possuem conhecimentos científicos acerca das suas atividades biológicas, resultando por vezes, em efeitos adversos. Considerações finais: Apesar do uso do gênero para fins terapêuticos se mostrar bastante difundido, em algumas situações essa prática pode não ser recomendada, devido a toxicidade que permeia as substâncias presentes, fazendo-se necessários estudos de toxicidade de modo a estimar a segurança bem como os verdadeiros potenciais terapêuticos.

Palavras-chave: Plantas medicinais, Fitoterapia, Crescentia, Toxicidade.

\section{ABSTRACT}

Objective: To review and compile information described in the literature on the chemical composition and biological activities of the genus Crescentia. Bibliographic review: Crescentia cujete L. proved to be as effective as Crescentia alata kunt in terms of therapeutic effects. The species have several bioactive compounds isolated and identified through spectrometric techniques, these compounds, linked to the biological activities presented in the species of the genus, even those activities that require relative toxicity, such as antimicrobial and anticancer activities, standing out as a future candidate to supply its components to the pharmaceutical industry. Many species are used, in popular use, inappropriately, considering that most users do not have scientific knowledge about their biological activities, sometimes resulting in adverse effects. Final considerations: Although the use of the genus for therapeutic purposes is widespread, in some situations this practice may not be recommended, due to the toxicity that permeates the present substances, making toxicity studies necessary in order to estimate safety as well as the true therapeutic potentials.

Key words: Medicinal plants, Phytotherapy, Crescentia, Toxicity.

\section{RESUMEN}

Objetivo: Revisar y compilar la información descrita en la literatura sobre la composición química y las actividades biológicas del género Crescentia. Revisión bibliográfica: Crescentia cujete L. demostró ser tan eficaz como Crescentia alata kunt en términos de efectos terapéuticos. Las especies tienen varios compuestos bioactivos aislados e identificados mediante técnicas espectrométricas, estos compuestos, vinculados a las actividades biológicas presentadas en las especies del género, incluso aquellas actividades que requieren toxicidad relativa, como las actividades antimicrobianas y anticancerígenas, se destacan como un futuro candidato para suministrar sus componentes a la industria farmacéutica. Muchas especies se usan, en uso popular, de manera inapropiada, considerando que la mayoría de los usuarios no tienen conocimiento

\footnotetext{
1 Faculdade de Integração do Sertão (FIS), Serra Talhada - PE. *E-mail: limmavivianne@gmail.com

2 Instituto de Ciência, Tecnologia e Qualidade (ICTQ), Juazeiro do Norte - PE.

${ }^{3}$ Universidade Federal de Pernambuco (UFPE), Recife - PE.
} 
científico sobre sus actividades biológicas, lo que a veces produce efectos adversos. Consideraciones finales: Aunque el uso del género con fines terapéuticos es generalizado, en algunas situaciones esta práctica puede no recomendarse, debido a la toxicidad que impregna las sustancias presentes, lo que hace necesarios estudios de toxicidad para estimar la seguridad y Los verdaderos potenciales terapéuticos.

Palabras clave: Plantas medicinales, Fitoterapia, Crescentia, Toxicidad.

\section{INTRODUÇÃO}

O uso de plantas medicinais para prevenção e recuperação da saúde é considerada uma prática que atravessa milênios, com origem na China e Egito, difundindo-se, posteriormente, para resto do mundo, e historicamente presente na sabedoria empírica, desde as mais simples até as mais elaboradas formas de uso terapêutico. Com o decorrer do tempo, a medida que as populações utilizavam a biodiversidade disponível, as plantas foram classificadas e selecionadas, originando técnicas de cultivo para diversos fins, articulando cultura e saúde. Nesse aspecto, além de sua importância biológica, os recursos vegetais também têm grande importância biocultural (MARTINS MC e GARLET TMB, 2016; RODRIGUES TA, et al., 2020; VIEIRA-FILHO MAM, et al., 2018).

Esse potencial terapêutico é demostrado através da fitoterapia e dos inúmeros medicamentos derivados de espécies vegetais. A fitoquímica possui grande relevância para o desenvolvimento científico dos produtos naturais, contribuindo ainda para o avanço de outras atuações científicas. Tal estudo engloba a elucidação dos produtos originários do metabolismo secundário dos vegetais, compreendendo as etapas de purificação, isolamento e definição estrutural dos metabólitos. Os vegetais possuem inúmeras substâncias químicas que podem apresentar atividades biológicas, constituindo um recurso terapêutico relevante, pois seus princípios ativos são introduzidos em fármacos no mundo inteiro (ALMEIDA-CINCOTTO MGJ, et al., 2016).

A Bignoniaceae trata-se de uma família vegetal pantropical, com poucos representantes nas regiões temperadas. Atualmente são reconhecidas 840 espécies de plantas arbustivas, arbóreas e trepadeiras na família, cerca de $80 \%$ (660 spp.) ocorrem na região neotropical e quase metade (407 spp.) no Brasil, distribuída em todo o Nordeste. Contém diversos componentes químicos, tais como, os flavonoides, terpenóides, quinonas, mas principalmente nafthoquinonas e compostos aromáticos, sendo que o gênero Crescentia está entre os membros mais importantes da família devido suas consideráveis propriedades medicinais (LOHMANN LG, et al., 2018; GÓMEZ OC e LUIZ JHH, 2018).

No gênero, destaca-se a Crescentia cujete L., uma espécie neotropical, conhecida popularmente como coité, cuieira, cabeceira ou árvore de cabaça. No Brasil, não possui um habitat único, plantas da família Bignoniaceae ocorrem no Cerrado, Mata Atlântica, região Amazônica e Caatinga, onde grande parte das espécies são encontradas em cerrados e caatingas por discorrer áreas de vegetação aberta (CAMARINHA C, et al., 2015).

Considerando que são escassos os estudos que confirmem as atividades biológicas e toxicidade desta espécie, o objetivo do presente estudo é produzir uma compilação de informações descritas na literatura, reunindo dados científicos que possam contribuir como direcionamento para pesquisas futuras sobre a composição química e atividades biológicas do gênero.

\section{REVISÃO BIBLIOGRÁFICA}

Estima-se que $40 \%$ da economia mundial baseia-se em produtos e processos relacionados a biodiversidade. Setores madeireiros, alimentícios, industrias de medicamentos e cosméticos utilizam essa variedade biológica como fonte de matérias-primas (PIMENTEL V, et al., 2015).

A medicina apresenta diversos compostos extraídos de recursos naturais, com destaque para bioativos presentes nas plantas. Exemplo clássico é a Morfina, fármaco narcótico de alto poder analgésico, extraído a partir do extrato de folhas de papoula (Papaver somniferum). No início do século XIX, houve amplificação significativa das propriedades analgésicas dessa planta, que já era conhecida desde o Império Romano, ao longo dos séculos, outros compostos foram extraídos de plantas, sendo empregados até hoje, como codeína, efedrina e quinina (KINGSTON DGI, 2011). 
No uso popular, muitas espécies são utilizadas sem que haja estudos toxicológicos. Na crença de que não possuem efeitos colaterais, em grandes centros urbanos, parte da comercialização de produtos fitoterápicos é feita em mercados e feiras populares, com fiscalização pouco eficiente (RODRIGUES E, et al., 2010). Dessa forma, estudos relacionados à composição química podem contribuir de modo efetivo na produção de novas drogas com segurança e eficácia.

Mendieta MC et al. (2014) descrevem em seu estudo que as plantas apresentam substâncias que podem desencadear reações adversas, seja por seus próprios componentes, ou então se tornam perigosas, devido a coleta ou extração errônea de seus constituintes. Algumas plantas tóxicas são tidas como ornamentais, logo estando presente em diversos ambientes de nosso cotidiano, por conseguinte facilitando 0 risco de intoxicação. Dados apontam que apenas cerca de $5 \%$ destas plantas têm sido avaliadas fitoquimicamente e sobre os aspectos biológicos apresenta um percentual ainda menor (BARREIRO EJ et al., 2009).

No uso popular, muitas espécies são utilizadas sem que haja estudos toxicológicos. Na crença de que não possuem efeitos colaterais, em grandes centros urbanos, parte da comercialização de produtos fitoterápicos é feita em mercados e feiras populares, com fiscalização pouco eficiente (RODRIGUES E et al., 2010). Dessa forma, estudos relacionados à composição química podem contribuir de modo efetivo na produção de novas drogas com segurança e eficácia.

\section{Aspectos botânicos do gênero Crescentia}

$\mathrm{Na}$ família Bignoniaceae, o gênero Crescentia possui grande expressão, há cerca de seis espécies, bastante cultivadas devido ao seu valor ornamental, em razão do formato de seus frutos (MADHUKAR VK, et al., 2013). Dentre elas a Crescentia cujete L., amplamente cultivada em toda a região neotropical desde os tempos pré-histórico, Crescentia alata K. que ocorre desde o México até a Costa Rica, Crescentia. amazonica Ducke na Amazônia e no alto do rio Orinoco, Crescentia mirabilis e Crescentia portoricensis são restritas às ilhas do Caribe e Crescentia linearifolia a qual é amplamente distribuída no Caribe (ARANGO-ULLOA JA, et al., 2009; AGUIRRE-DUGUA X, et al., 2012).

Porém pesquisas com espécies do gênero ainda são escassas, sendo encontrados estudos biológicos, bem como fitoquímicos de apenas duas espécies do gênero, a Crescentia alata Kunt e a Crescentia cujete L., comprovando assim a carência de estudos sobre o gênero. A literatura descreve a Crescentia alata Kunt como uma espécie frutífera tropical. Na medicina popular são usadas, flores, cascas do caule, folhas e frutas, no tratamento de diversas doenças, no entanto, sua eficácia a nível científico ainda é pouco conhecida, encontrada com maior expressão no México chegando até a Costa Rica (AGUIRRE-DUGUA X, et al., 2018).

As espécies desse gênero são utilizadas para diversos fins, como na horticultura, na construção civil, obtenção de tintas, carpintaria e construção de instrumentos musicais e no urbanismo devido a beleza de seus frutos e florações para paisagismo. As flores do gênero são diversas em relação a forma, cor e fase de antese. Sua polinização é cruzada, promovida pela vinda de estigma sensitivo, e as sementes na maior parte das espécies são dispersas pelo vento. Os polinizadores são as abelhas, borboletas, mariposas, vespas, morcegos e aves (MARTIN F et al., 2007).

A Crescentia cujete $L$. é a espécie mais explorada do gênero, amplamente cultivada em na região neotropical desde os tempos pré-históricos, no Brasil é conhecida popularmente como "árvore de cabaça", "coité", "cuieira" ou "cabaça" (AZEVEDO CF, et al., 2010). Configura-se como árvores de pequeno porte de aproximadamente 5 a $10 \mathrm{~m}$ de altura, pode apresentar tanto hábito arbóreo, lianescente ou arbustivo com cálice bipartido, flores verdes amarelada, as quais florescem de julho a setembro e seus frutos ocorrem de setembro a outubro (AGUIRRE-DUGUA X, et al., 2018). Ilustra folhas bifurcadas de coloração verde escuro, fruto duro de formatos esféricos, ovoides ou globosos, chegando até 25 centímetros, com polpa gelatinosa e numerosas sementes, quando secos são comumente usados na confecção de artesanatos, recipientes e utensílios domésticos que a inclui entre as plantas ornamentais (PEREIRA SG, et al., 2015).

No sul do México foram encontradas populações silvestres que apresentam frutos alongados, pequenos e com exocarpo mais fino que os tipos cultivados nos países vizinhos, alta diversidade morfológica também foi registrada nas áreas caribenhas e da Bacia do rio Orinoco na Colômbia. Dos seis principais biomas brasileiros: Amazônia, Mata Atlântica, Cerrado, Caatinga, Pantanal e Pampa, a Mata Atlântica e Nordeste 
possuem a maior diversidade de espécies. Enquanto, o Pantanal e Pampa configuram os menores números de representantes (ARANGO-ULLOA JA, et al., 2009; AGUIRRE-DUGUA X, et al., 2012; LOHMANN LG, 2018).

Como evidenciado por Ejelonu BC et al. (2011) a espécie é largamente utilizada como ornamental, se cultivadas em jardins, atuam como cercas-vivas, sendo muito usada em praças, jardins públicos e na arborização de ruas. Devido seu formato e resistência, os frutos secos após a remoção da polpa, são comumente usados na confecção de tigelas conhecidas popularmente como cuias, recipientes e utensílios domésticos, destacando que a seiva extraída da planta pode ser utilizada como inseticida para pragas; as sementes fornecem um óleo fixo artesanal muito semelhante ao do azeite de oliva e amendoim.

\section{Aspectos fitoquímicos do gênero Crescentia}

Prospecções fitoquímicas e atividades biológicas de Crescentia alata Kunt também foram citados na literatura. Arenas FS (2004) em seu estudo identificou alguns grupos químicos presente nos frutos e folhas da Crescentia alata Kunt, derivados como epigenina e quercitina, do grupo flavonóide, podendo ser responsáveis pela atividade anti-inflamatória, assim como as furanoaftoquinonas são possíveis mediadoras da atividade anticancerígena relatadas na espécie.

González-Gómez JC et al. (2006) notou a presença de fenóis totais e taninos condensados na $C$. alata Kunt, e pode observar que a esta possui baixa concentrações desses compostos. Análises moleculares baseadas em bandas polimórficas de marcadores (AFLP) polimorfismo de comprimento de fragmento amplificado, apontaram que a Crescentia alata Kunt é espécie mais similar a Crescentia cujete, considerando que a Crescentia alata Kunt pode ser uma espécie híbrida na família (ARANGO-ULLOA JA, et al., 2009).

Autore $\mathrm{G}$ et al. (2001) avaliou o extrato metanólico das folhas de $C$. alata, e conseguiram isolar três flavonoides, sendo dois glicosilados, sendo que um deles, o kaempferol, mostrou atividade anti-inflamatória in vitro na proteção induzida pela endotoxina em macrófagos J774A1 que inibem diretamente a via L-argininaNO.

Em outra pesquisa utilizando o extrato metanólico dos frutos maduros de $C$. alata, foram isolados quatro terpenos da classe dos iridoides, considerados como os principais responsáveis pela atividade larvicida contra Spodoptera frugiperda. Foi observado que quatro frações produziram mortalidade de $(40 \%)$ a $(80 \%)$, devido principalmente aos iridoides, que são os principais constituintes de $C$. alata, mostrando que a espécie possui potencial para ser considerada como um forte bioinsecticida (VALLADARES-CISNEROS MG, et al., 2014).

Alguns compostos bioativos de Crescentia cujete foram isolados e identificados através de técnicas espectrométricas, como triagens fitoquímicas preliminar e cromatográfica. Macedo WA et al. (2018) relatam diversos compostos presentem no extrato das folhas, os principais são as naftoquinonas, glicosídeos iridóides, plumierida, aucubina e asperuloside. Martins AML e Almeida SSMS (2012), em seu estudo também puderam observaram a presença de metabólitos importantes como, ácidos orgânicos, saponinas, fenóis e taninos.

Os ácidos orgânicos atuam como anti-inflamatório e antiespasmódicos, os taninos têm ação bactericida e fungicida, já as saponinas têm atividade antimicrobiana e espermicida. Antraquinonas e cardenolídeos também foram evidenciados, quando isolados ou extraídos na forma de um fitocomplexo, podem ser utilizados na produção de fitofármacos (EJELONU BC, et al., 2011; KUSTER VC, et al., 2016). Coe FG et al. (2012) relatam ausência de alcaloides na espécie. Entretanto, a eficácia de $C$. cujete nas atividades biológicas descritas na literatura é relacionada à presença de outros tipos de metabolitos secundários, tais como os triterpenos da classe dos iridoides, flavonoides, glicosídeos, fenóis, saponinas, esteroides e taninos.

A investigação química dos frutos levou ao isolamento de uma série de glicosídeos, iridóides, iridóides glicosilados, alquil glicosídeos, glicose p-hidroxi benzoiloxi, 11-noriridóides, ácidos orgânicos e alguns derivados aromáticos. A polpa da $C$. cujete, na medicina veterinária, além de ser empregada em dermatites, queimaduras solares, possui propriedades repelentes contra pulgas (ARRUDA MMS, et al., 2011). A ação antifúngica pode estar relacionada a presença de flavonóides, fenóis, saponinas e terpenos, todos já relatados como presente em extratos do fruto. 


\section{Atividades biológicas embasadas cientificamente}

O extrato metanólico das folhas de $C$. alata foi testado para atividade anti-inflamatória in vivo sobre edema de pata de rato induzido por carragenina, e demostrou uma significativa atividade anti-inflamatória a dose testada (500 mg/kg) considerada uma dose mais elevada (AUTORE G, et al., 2001). As cascas do caule de C. alata kunt foram estudadas por Rodriguez-Lopez V et al. (2003) testando a atividade espasmolítica sobre as contrações espontâneas em íleo isolado de rato, e não obteve atividade satisfatória no estudo.

Do ponto de vista etnofarmacológico, a Crescentia cujete é frequentemente utilizada no controle da hipertensão (MARTINS AML e ALMEIDA SSMS, 2012), no tratamento de hematomas e tumores, atua como analgésico, expectorante, antitussígeno e como medida paliativa para problemas respiratórios (AZEVEDO FC, et al., 2010) também utilizada no tratamento de alguns distúrbios ginecológicos. O chá das folhas, através da infusão ou maceração, é utilizado no tratamento da diabetes, tosses, anemias, além de hemorragias causada por picada de animais peçonhentos (SHASTRY CS, et al., 2012).

O chá das folhas, através da infusão ou maceração, é utilizado no tratamento da diabetes, tosses, anemias, além de hemorragias causada por picada de animais peçonhentos. A decocção das raízes é utilizada como antiparasitário, no tratamento de amebíase, as cascas são usadas para a limpeza de feridas e preparação de cataplasma. Mostrou atividade antibacteriana contra bactérias gram-positiva e gram-negativa e ação antifúngica, sendo capaz de inibir a levedura Candida albicans (EJELONU BC, et al., 2011; PARVIN MS, et al., 2015; ROJAS G et al., 2001; SHASTRY CS, et al., 2012).

Os efeitos fitotóxicos dos frutos foram avaliados em ensaios de germinação das sementes das plantas invasoras Mimosa pudica Mill., Senna obtusifolia L., o extrato aquoso bruto nas concentrações testadas não apresentou citotoxicidade.

No entanto, a fase orgânica da C. Cujete L., inibiu 100\% a germinação das sementes das duas espécies receptoras, obtendo o efeito citotóxico na concentração de $0,5 \%$, considerada relativamente baixa (PEREIRA MS, et al., 2015). Macedo WA, et al. (2018) concluíram em estudo que a espécie pode ser tóxica ao organismo humano e animal, em seus ensaios pode observar que houve presença de anomalias e baixo índice mitótico ocasionando baixa divisão celular em concentrações mais elevadas.

No estudo foram testaram diferentes concentrações, e foi possível observar que o menor índice mitótico foi nos tempos de 24 e 48 horas, tendo em vista que o tratamento de 0,45 gramas/L no tempo de 24 horas apresentou maior índice mitótico. Outro estudo foi realizado oferecendo polpa da fruta de $C$. cujete a caprinos por vários dias consecutivos relataram que a espécie tem grande potencial abortivo e que é uma causa esporádica de abortos em ruminantes (ASSIS TS, et al., 2009).

Bussmann RW et al. (2011) e Coe FG et al. (2012) confirmam toxicidade da espécie, contra Artemia salina e larvas de Aedes aegypti, em estágios III e IV, no qual seus extratos das folhas, aquoso e alcoólico, foram considerados como altamente tóxicos, evidenciando diferentes $\mathrm{CL}_{50}$ de acordo com o tipo de extrato, em um dos estudos, o etanólico apresentou maior potencial citotóxico em relação ao aquoso, exibindo CL50 $0,74 \mu \mathrm{g} / \mathrm{mL}$ e $15 \mu \mathrm{g} / \mathrm{mL}$, respectivamente, revelando uma provável afinidade dos compostos com meios extratores apolares. Já no estudo de Espitia-Baena GE, et al. (2011) ele observou que não foram produzidos efeitos tóxicos em larvas de Artemia salina e Aedes aegypti, utilizando o extrato das cascas do caule de $C$. cujete. Essa discrepância na atividade toxicológica pode ser explicada pela presença ou ausência de alguns metabólitos secundários ou concentração variada de compostos tóxicos.

A maioria dos estudos relacionados às atividades biológicas de extratos de Crescentia cujete $L$. consistiram em avaliar a atividade antimicrobiana. Agarwal M e Chauhan S (2015) em seu estudo confirmam a correlação dos usos relatados da Crescentia cujete na medicina tradicional agentes infecciosos, corroborando com estudo de Parvin MS et al. (2015) que demonstrou atividade antimicrobiana dos extratos aquosos e alcoólicos de casca de caule e folhas de Crescentia cujete. Através do método de difusão em disco, foi possível observar a inibição de Staphylococcus aureus e Escherichia coli. Porém ocorre diferença na atividade antimicrobiana dependendo do tipo de meio extrator utilizado para uma mesma parte da planta (UGBABE GE, et al., 2010).

REAS/EJCH | Vol.12(9) | e3886 | DOI: https://doi.org/10.25248/reas.e3886.2020 Página 5 de $\mathbf{7}$ 
O extrato etanólico dos frutos e folhas foi citado como agente anti-hemorrágico, mostrando grande capacidade de neutralizar efeito hemorrágico provocados por acidentes ofídicos (cobras venenosas), o extrato foi testado frente o veneno das serpentes Bothrops atrox e Vipera russelli, sendo ativo apenas na neutralização do veneno de $V$. russelli. Tais resultados demonstram a capacidade do extrato em antagonizar o veneno, que pode ser justificada pela presença de compostos fenólicos, como taninos e flavonóides, que possuem ação anti-hemorrágica (SHASTRY CS, et al., 2012).

Das N, et al. (2014) e Lizcano LJ, et al. (2010) em suas pesquisas relatam testes para avaliação da atividade antioxidante nas folhas e frutos de $C$. cujete, através dos métodos do sequestro do radical livre 2,2difenil-1-picrilhidrazil (DPPH), pela capacidade de absorção do radical oxigênio (CARO), poder redutor férrico (PRF), e capacidade antioxidante equivalente do Trolox (CAET). As folhas possuem significativas propriedades de eliminação de radicais livres pelos métodos do DPPH e PRF. No entanto, para os métodos CARO e CAET, o extrato avaliado dos frutos não mostrou atividade.

A ação anti-inflamatória do extrato etanólico das folhas e das cascas do caule foi avaliada através do método in vitro de estabilização de glóbulos vermelhos no sangue humano, no qual foi observado potencial para estabilização das membranas. A atividade anti-inflamatória pode estar relacionada com a presença dos derivados da quercetina e apigenina, compostos fenólicos conhecidos por apresentar elevada capacidade anti-inflamatória, e foram isolados nas folhas da espécie em estudo. O extrato das cascas do caule e das folhas de $C$. cujete $L$. foram avaliados para a atividade anti-inflamatória através do método in vitro de estabilização de glóbulos vemelhos do sangue humano, e foi notado alto potencial na estabilização da membrana (PARVIN MS, et al., 2015).

\section{CONSIDERAÇÕES FINAIS}

O gênero Crescentia como instrumento de pesquisa, mostrou-se bastante proeminente em relação aos efeitos farmacológicos discutidos, destacando-se as espécies Crescentia cujete $L$. e Crescentia alata kunt como futuras candidatas a fornecer seus componentes para a indústria farmacêutica. A prospecção química e atividades biológicas do gênero Crescentia relatadas na literatura, estão de acordo com conhecimento popular apresentado das espécies. Porém, estudos sobre o potencial tóxico deste gênero ainda são incipientes, evidenciando a necessidade da realização de novas pesquisas que possam contribuir como subsídios para novos ensaios toxicológicos do gênero.

\section{REFERÊNCIAS}

1. AGARWAL M, CHAUHAN S. Anti-Mycobaterial Potential of Crescentia cujete (Bignoniaceae). International Journal of Advanced Research in Botany (IJARB), 2015; 1:1-9.

2. AGUIRRE-DUGUA X, et al. Round and large: morphological and genetic consequences of artificial selection on the gourd tree Crescentia cujete by the Maya of the Yucatan Peninsula, Mexico. Annals of Botany, 2012; 109(1):12971306.

3. AGUIRRE-DUGUA $X$, et al. Anthropogenic dispersion of selected germplasm creates a geographic mosaic of contrasting maternal lineages in Crescentia cujete from Mesoamerica. Tree Genetics \& Genomes, 2018; $14(2): 1-18$.

4. ALMEIDA-CINCOTTO MGJ, et al. Avaliação do potencial citotóxico e do potencial em estimular a biossíntese de colágeno de extrato de folhas de Morus nigra L. em cultura celular. Rev. Ciên. Farm. Básica Apl.,2016; 37(1):1-1.

5. ARENAS FS. Etnobotánica y usos potenciales del cirián (Crescentia alata, h.b.k.) en el estado de morelos. Polibotanica, México., 2004; 18:13-31.

6. ARRUDA MMS, et al. Estudo preliminar do extrato etanólico de polpa dos frutos de Crescentia cujete L. (Bignoniaceae) sobre larvas de Rhipicephalus (Boophilus) microplus. In: 34ª Reunião Anual da Sociedade Brasileira de Química. 2011.

7. ASSIS TS, et al. Intoxicações por plantas em ruminantes e equídeos no Sertão Paraibano Plant poisonings in ruminants and equidae in the Sertão of Paraiba, Brazil. Pesquisa Veterinária Brasileira, 2009; 29(1): 919-924.

8. AUTORE G, et al. Inhibition of nitric oxide synthase expression by a methanolic extract of Crescentia alata and its derived flavonols. Life Sci, 2001; 70(5): 523-534.

9. AZEVEDO CF, et al. Germinação de sementes de cabaça em diferentes substratos e temperaturas. Revista Brasileira de Ciências Agrárias, 2010; 5(3):354-357.

10. BARREIRO EJ, BOLZANI VS. Biodiversidade: fonte potencial para a descoberta de fármacos. Química Nova, São Paulo, 2009;32(3):679-688.

11. BUSSMANN RW. et al. Toxicity of medicinal plants used in traditional medicine in Northern Peru. J. Ethnopharmacol., 2011; 137(1): 121-140. 
12. CAMARINHA C, et al. Levantamento de espécies da família Bignonniaceae ocorrentes na universidade de Castelo Branco, Campus Realengo - RJ. Revista Eletrônica de Biologia, 2015; 8 (3): 299-307.

13. COE FG, et al. The good and the bad: Alkaloid screening and brineshrimp bioassays of aqueous extracts of 31 medicinal plants of eastern Nicaragua. Pharm Biol, 2012; 50(3): 384-392.

14. DAS N, et al. Antioxidant activities of ethanol extracts and fractions of Crescentia cujete leaves and stem bark and the involvement of phenolic compounds. BMC Compl Alt Med, 2014; 14(1): 1-45.

15. EJELONU BC, et al. The chemical constituents of calabash (Crescentia cujete). African Journal of Biotechnology, 2011; 10(4): 19631-19636.

16. ESPITIA-BAENA JE, et al. Química y biología del extracto etanólico del epicarpio de Crescentia cujete L. (totumo). Rev Cub Plant Med, 2011; 16(4): 337-346.

17. GÓMEZ OC, LUIZ JHH. Endophytic fungi isolated from medicinal plants: future prospects of bioactive natural products from Tabebuia/Handroanthus endophytes. Applied microbiology and biotechnology, 2018; 5:1-15.

18. GONZÁLEZ-GÓMEZ JC, et al. Determinação de fenóis totais e taninos condensados em espécies arbóreas com potencial forrageiro na região Tierra Caliente de Michoacán, México. Pesquisa Pecuária para o Desenvolvimento Rural, 2006; 18(11):1-15.

19. KINGSTON DGI. Modern Natural Products Drug Discovery and Its Relevance to Biodiversity Conservation. Journal of Natural Products, Estados Unidos, 2011; 74:496-511

20. KUSTER VC, VALE FHA. Leaf histochemistry analysis of four medicinal species from Cerrado. Revista Brasileira de Farmacognosia, 2016; 26 (6): 673-678.

21. LIZCANO LJ, et al Antioxidant activity and polyphenol content of aqueous extracts from Colombian Amazonian plants with medicinal use. Food Chem, 2010; 119(4): 1566-1570.

22. LOHMANN LG, et al. Flora das cangas da Serra dos Carajás, Pará, Brasil: Bignoniaceae. Rodriguésia. 2018; 69(3):1063-1079.

23. MACEDO WA, et al. Efeito citotóxico e genotóxico de crescentia cujete I. (bignociaceae) através do bioteste Allium cepa. Centro Científico Conhecer - Goiânia, 2018; 5(10): 66-73.

24. MADHUKAR VK, et al. Revision of Genus Crescentia L. (Bignoniaceae) in India, American Journal of Plant Sciences, 2013;4(6):1-5.

25. MARTIN F, et al. Iridoid glycosides from the stems of Pithecoctenium crucigerum (Bignoniaceae). Phytochem, 2007; 68 (9):1307-1311.

26. MARTINS AML, ALMEIDA SSMS. Estudo fitoquímico da poupa de Crescentia cujete I. (Bignoniaceae). Livro de resumos do $3^{\circ}$ Congresso amapaense de iniciação científica e $3^{\circ}$ Exposição de pesquisa científica. 2012.

27. MARTINS MC, GARLET TMB. Desenvolvendo e divulgando o conhecimento sobre plantas medicinais. Revista Eletrônica em Gestão, Educação e Tecnologia Ambiental, 2016; 20(1):438-448.

28. MENDIETA MC, et al. Plantas tóxicas: importância do conhecimento para realização da educação em saúde. Revista de enfermagem, UFPE on line., Recife, 2014; 8 (3):680-686.

29. PARVIN MS, et al. Evaluation of in vitro anti-inflammatory and antibacterial potential of Crescentia cujete leaves and stem bark. BMC Research Notes, 2015; 8(1):412.

30. PIMENTEL V, et al. Biodiversidade brasileira como fonte da inovação farmacêutica: uma nova esperança? Revista do BNDES, 2015; 43:41-89.

31. RODRIGUES E, et al. Perfil farmacológico e fitoquímico de plantas indicadas pelos caboclos do Parque Nacional do Jaú (AM) como potenciais analgésicas. Parte I. Revista Brasileira de Farmacognosia, 2010; 20(6):981-991.

32. RODRIGUES TA, et al. valorização das plantas medicinais como alternativa à saúde: um estudo etnobotânico. Revista Ibero Americana de Ciências Ambientais, 2020; 11(1):411-428.

33. RODRIGUEZ-LOPEZ V, et al. Spasmolytic activity of several extracts obtained from some Mexican medicinal plants. Fitoterapia, 2003; 74(7):725-728.

34. ROJAS G, et al. Antimicrobial evaluation of certain plants used in Mexican traditional medicine for the treatment of respiratory diseases. J Ethnopharm, 2001; 74(1): 97-101.

35. SHASTRY CS, et al. Antivenom activity of ethanolic extract of Crescentia cujete fruit. Int J Phytomed, 2012; 4(1): 1108.

36. UGBABE GE. et al. Preliminary Phytochemical and Antimicrobial Analyses of the Leaves of Nigerian Bignoniaceae Juss. Global Research Journals, 2010; 1:1-5.

37. VALLADARES-CISNEROS MG, et al. Biological Activity of Crescentia alata (Lamiales: Bignoniaceae) Fractions on Larvae of Spodoptera frugiperda (Lepidoptera: Noctuidae). Florida Entomologist, 2014; 97(2):770-777.

38. VIEIRA-FILHO MAM, et al. Diversidad biocultural asociada al uso actual de plantas medicinales em uma comunidade rural en el litoral piauiense (Noreste de Brasil). Ethnoscientia, 2018; 3:1-13. 